Check for updates

Cite this: RSC Adv., 2018, 8, 9344

\title{
Electrospun nanofiber templated assembly of hybrid nanoparticles $\uparrow$
}

\begin{abstract}
Zhicheng Liu, ${ }^{\text {ac }}$ Zhaodong Yan ${ }^{a}$ and Lu Bai $\mathbb{D}$ *b
Assembling nanoparticles into or onto a three-dimensional template such as an electrospun nanofiber membrane has attracted considerable attention since this composite material has great potential in many applications. We report here that hybrid noble metal nanoparticles could be readily assembled both into and onto electrospun nanofibers using simple mixing and immersion steps. It is observed that small gold nanospheres were well distributed within the nanofiber, while other nanoparticles such as big gold nanospheres, gold nanorods and palladium nanocubes were uniformly decorated on the surface of the nanofibers. Moreover, the hybrid nanoparticle-assembled nanofiber membrane showed impressive SERS and catalytic performance based on the type of the assembled nanoparticles. It is believed that other nanomaterials could also be assembled with nanofiber membranes using this facile strategy.
\end{abstract}

Received 22nd January 2018 Accepted 28th February 2018

DOI: $10.1039 / \mathrm{c} 8 \mathrm{ra00665b}$

rsc.li/rsc-advances hand, the NPs are protected by the surrounding polymers from aggregation, and on the other hand, the chemicals or analytes should diffuse through the polymer barrier to access the active NPs. In other cases, NMNPs could be directly assembled onto the surface of electrospun nanofibers through various kinds of interactions such as electrostatic interaction and hydrogen bonding. ${ }^{14-18}$ For instance, by taking advantage of the hydrogen bonding interaction between the amide groups on the nanofibers and the carboxylic acid groups on the NPs, Au NPs were successfully decorated on the surface of the polyamide nanofiber. ${ }^{14}$ It is worth mentioning that there are few reports showing the assembly of two or more kinds of NMNPs into or onto electrospun nanofibers, although there might be new or improved properties because of the increased NP loading and the possible synergistic effect. Recently, Zhang and Yu presented that Au NRs could be assembled on the Ag nanowires (NWs) to form an Au NR-Ag NW nanocomposite. ${ }^{19}$ Then the nanocomposites were electrospun into nanofiber membranes with different optical properties and enhanced SERS activity. Given that little effort has been made in this subject, it remains a challenge to design and fabricate 3D electrospun nanofiber membranes assembled with different kinds of NMNPs.

In this work, composite nanofiber membranes with diverse metal NPs assembled both into and onto the nanofibers were facilely prepared. Firstly, small Au NPs were assembled inside the electrospun poly(acrylic acid)/poly(vinyl alcohol) (PAA/PVA) nanofibers by mixing the Au NP solution with the polymer solution. Then, driven by electrostatic interaction, both spherelike and anisotropic positively-charged NMNPs were immobilized outside the nanofiber through a simple immersion step. Moreover, due to the high loading of NMNPs, these 3D NPsassembled nanofiber membranes showed excellent SERS and catalytic property.
${ }^{a}$ School of Materials Science and Engineering, North University of China, Taiyuan 030051, China

${ }^{b}$ School of Chemical Engineering and Technology, North University of China, Taiyuan 030051, China. E-mail: bailu0919@gmail.com

${ }^{c}$ Department of Mechanical Engineering, National University of Singapore, Singapore, 117574, Singapore

$\dagger$ Electronic supplementary information (ESI) available. See DOI: 10.1039/c8ra00665b 


\section{Experimental}

\section{Materials}

PAA $\left(M_{\mathrm{w}}=240000\right)$ was obtained from J\&K Scientific (Beijing, China). PVA (PVA-2088) was purchased from Chenqi Chemical Technology Co., Ltd (Shanghai, China). Sodium borohydride $\left(\mathrm{NaBH}_{4}\right)$ was purchased from Macklin Biochemical Co., Ltd (Shanghai, China). Other chemicals such as 4-aminothiophenol (4-ATP) were from Sinopharm Chemical Reagent Co., Ltd (Shanghai, China). All chemicals were used as received without further purification.

\section{Synthesis of Au nanoparticles (Au NPs)}

Au NPs with different sizes were synthesized using the classical seeded growth method. ${ }^{20}$ The seed solution was prepared by adding $0.6 \mathrm{~mL}$ of ice-cold and freshly prepared $0.1 \mathrm{M}$ $\mathrm{NaBH}_{4}$ into $20 \mathrm{~mL}$ mixed solution of trisodium citrate $(2.5 \times$ $\left.10^{-4} \mathrm{M}\right)$ and chloroauric acid $\left(\mathrm{HAuCl}_{4}, 2.5 \times 10^{-4} \mathrm{M}\right)$. The color turned pink, suggesting the formation of Au seeds. To prepare the growth solution, $\mathrm{HAuCl}_{4}\left(2.5 \times 10^{-4} \mathrm{M}\right)$ and cetyltrimethylammonium bromide (CTAB, $0.08 \mathrm{M}$ ) were mixed in $100 \mathrm{~mL}$ aqueous solution, and $9 \mathrm{~mL}$ of the growth solution and $0.05 \mathrm{~mL}$ of $0.1 \mathrm{M}$ ascorbic acid solution were mixed. Subsequently, $1 \mathrm{~mL}$ of the seed solution was added under vigorously stirring. The mixed solution was used as seed solution for the next growth process in $30 \mathrm{~min}$. The small and big Au NPs (denoted as Au sNPs and Au bNPs) were finally obtained after one and two more similar growth processes, respectively.

\section{Synthesis of Pd nanocubes (Pd NCs)}

The CTAB-protected Pd NCs were synthesized as previously described. ${ }^{21}$ In brief, CTAB $(0.1820 \mathrm{~g})$ and sodium ascorbate $(0.0099 \mathrm{~g})$ were mixed in $15 \mathrm{~mL}$ of deionized water, and the solution was stirred at $50{ }^{\circ} \mathrm{C}$. Palladium nitrate dehydrate $(0.0108 \mathrm{~g})$ was dissolved in $5 \mathrm{~mL}$ of deionized water, and then those solutions were rapidly mixed together. The synthesis was completed in about $30 \mathrm{~min}$.

\section{Synthesis of Au nanorods (Au NRs)}

$\mathrm{Au}$ NRs were prepared according to the classic seed-mediated growth method. ${ }^{22}$ Firstly, $\mathrm{HAuCl}_{4}\left(2.5 \times 10^{-4} \mathrm{M}\right)$ and CTAB $(0.1 \mathrm{M})$ were mixed in $10 \mathrm{~mL}$ aqueous solution, and $0.6 \mathrm{~mL}$ of ice-cold and freshly prepared $0.1 \mathrm{M}$ aqueous $\mathrm{NaBH}_{4}$ solution were added. The resulted solution could be used as seed solution for the growth of Au NRs. Secondly, the growth solution was prepared by adding $2 \mathrm{~mL} 0.01 \mathrm{M} \mathrm{HAuCl}_{4}, 500 \mu \mathrm{L}$ $0.01 \mathrm{M} \mathrm{AgNO}_{3}$ and $300 \mu \mathrm{L} 0.1 \mathrm{M}$ ascorbic acid solution into

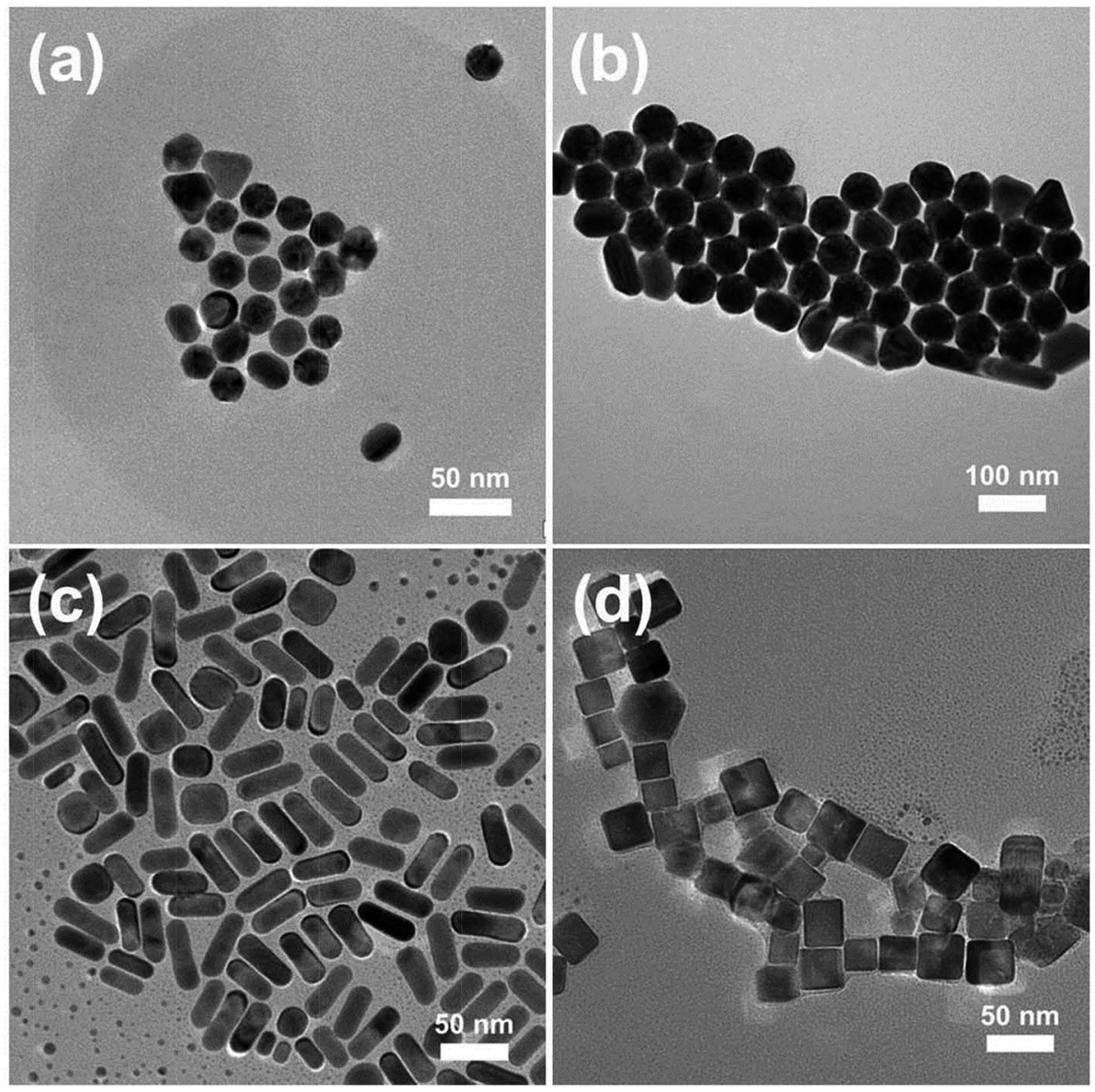

Fig. 1 TEM images of (a) Au sNPs, (b) Au bNPs, (c) Au NRs and (d) Pd NCs. 
$47.5 \mathrm{~mL} 0.1 \mathrm{M}$ CTAB aqueous solution. Finally, $60 \mu \mathrm{L}$ seed solution was added to the growth solution by hand shaking, and then the solution was left undisturbed overnight to grow Au NRs.

\section{Preparation of NMNPs assembled PAA/PVA electrospun} nanofibers

The Au sNPs were assembled inside the PAA/PVA electrospun nanofiber through simple solution blending. Briefly, the Au
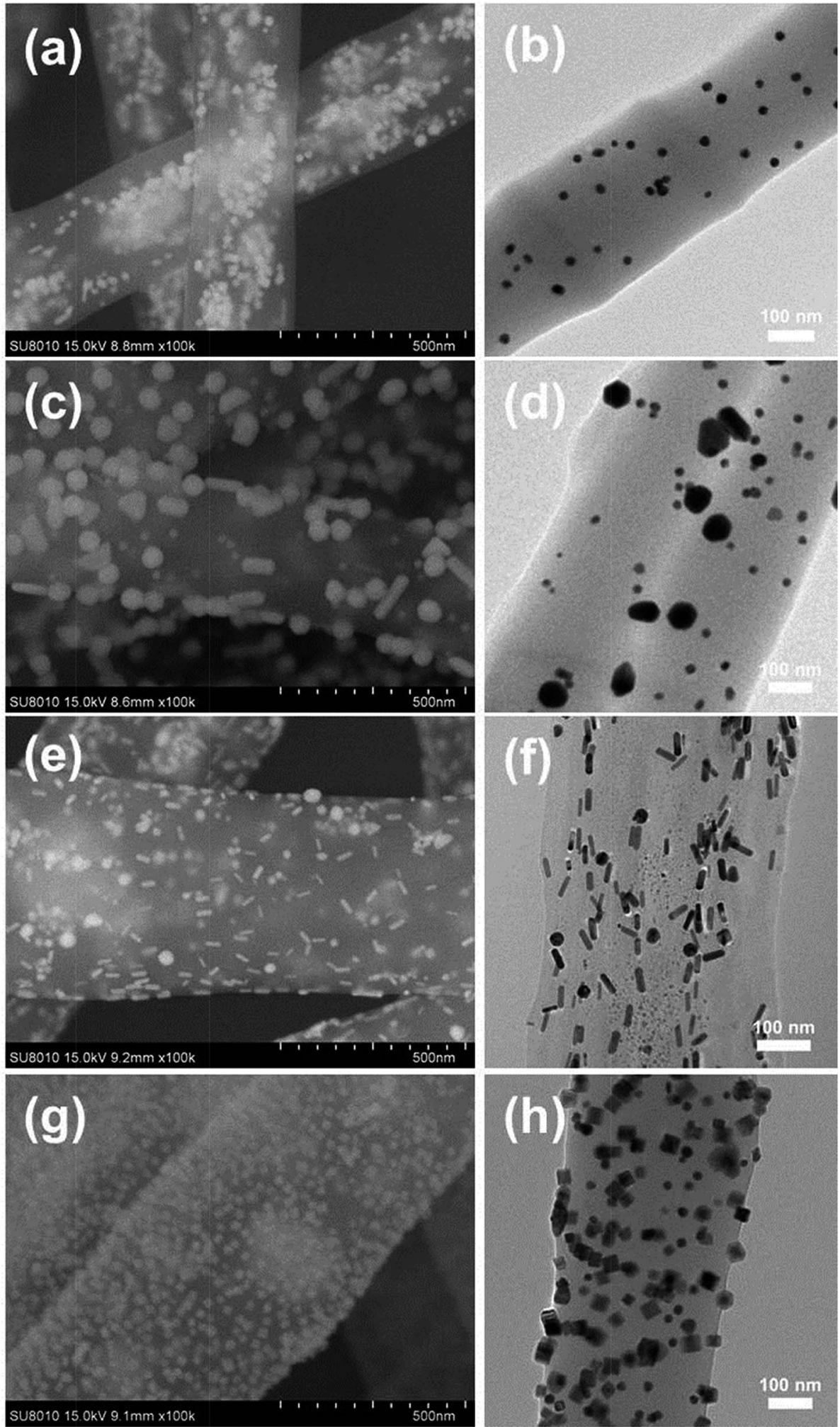

Fig. 2 SEM and TEM images of the PAA/PVA electrospun nanofibers assembled with (a and b) Au sNPs, (c and d) Au sNPs-Au bNPs, (e and f) Au sNPs-Au NRs and ( $g$ and h) Au sNPs-Pd NCs. 
SNPs solution was concentrated 100 -fold by centrifugation, and the solution was used to prepare a $10 \mathrm{wt} \%$ PAA/PVA solution. Then the hybrid solution was loaded into a syringe with a 22gauge blunt tip needle and electrospun into nanofibers. The applied voltage was $15 \mathrm{kV}$ while the flow rate was $300 \mu \mathrm{L} \mathrm{h}^{-1}$. The collection distance was $25 \mathrm{~cm}$. The electrospinning process was finished after $1.5 \mathrm{~h}$, and the resulted nanofibrous membrane was crosslinked by heat treatment at $145{ }^{\circ} \mathrm{C}$ for $30 \mathrm{~min}$ to produce water-stable nanofiber membrane. The membranes were immersed in the Au bNPs solution, the Au NRs solution and the Pd NCs solution for $12 \mathrm{~h}$ to assemble NMNPs, respectively. The membranes are also denoted as $\mathrm{Au}$ sNPs-Au bNPs, Au sNPs-Au NRs and Au sNPs-Pd NCs composite nanofiber membranes, respectively. Finally, these membranes were washed with water to remove the loosely bound NMNPs and left to dry in air.

\section{Catalytic reduction of 4-nitrophenol}

The catalytic activity of the Au sNPs-Pd NCs composite nanofibers toward 4-nitrophenol reduction was tested. A solution mixture containing 4-nitrophenol ( $5 \mathrm{~mL}, 1 \mathrm{mM})$ and $\mathrm{NaBH}_{4}$ aqueous solution $(5 \mathrm{~mL}, 0.1 \mathrm{M}$ ) was prepared in a $25 \mathrm{~mL}$ beaker. Then $35 \mathrm{mg}$ of the nanofibrous membrane was immersed into the solution at room temperature. The reaction rate of the 4nitrophenol reduction was evaluated using UV-vis spectroscopy.

\section{Characterization}

The NPs and the NPs-assembled nanofibers were observed using a transmission electron microscopy (TEM, JEOL-2100F) with an acceleration voltage of $200 \mathrm{kV}$. The morphologies of the NPs-assembled nanofibers were also observed by a scanning electron microscopy (SEM, Hitachi SU8010). The UV-vis absorption spectra were measured by a Unico UV-4800 UV-vis spectrometer. The X-ray diffraction (XRD) patterns were obtained from a DX-2700 X-ray diffractometer. The thermogravimetric (TG) experiments were performed on a TA Q 50 thermogravimetric analyzer. The metal content was measured using an inductively coupled plasma (Thermo Fisher Scientific, iCAP-6300) instrument. The SERS performance of the nanofibrous membranes was measured by a Renishaw InVia confocal Raman spectrometer using 4-aminophenol as probing molecule. All SERS spectra were recorded using $785 \mathrm{~nm}$ excitation, and the excitation power was $0.15 \mathrm{~mW}$. The integral time was $10 \mathrm{~s}$.

\section{Results and discussion}

The assembly of NPs both inside and outside the PAA/PVA nanofiber was achieved through a two-step assembly process. The first step is the mixing of the NP solution with the PAA/PVA solution to be electrospun, and the second step is the immersion of the NP assembled nanofibers into another NP solution. In order to assemble different kinds of NMNPs on 3D electrospun nanofiber membranes, Au NPs with different sizes, Au NRs as well as Pd NCs were synthesized. The typical TEM images of the CTAB-protected NPs and their UV-vis spectra are shown in
Fig. 1 and S1, $\uparrow$ respectively. The Au NPs (Fig. 1a and b) with diameters of about $23 \mathrm{~nm}$ (Au sNPs) and $62 \mathrm{~nm}$ (Au bNPs) were successfully obtained. The peaks of the UV-vis spectra located at $522 \mathrm{~nm}$ and $545 \mathrm{~nm}$ arise from the surface plasmon resonance of Au NPs. ${ }^{23}$ As shown in Fig. 1c, the as-synthesized Au NRs are about $46 \mathrm{~nm}$ in length and $17 \mathrm{~nm}$ in width (average aspect ratio was 2.7). The obvious absorption peaks at $520 \mathrm{~nm}$ and $730 \mathrm{~nm}$ (Fig. S1c $\dagger$ ) are typical peaks of Au NRs due to the well-known transverse and longitudinal plasmon resonance. ${ }^{23}$ Besides $\mathrm{Au}$ NRs, another kind of classic anisotropic NPs, i.e. Pd NCs were also prepared, as displayed in Fig. 1d. The cubical NPs had an edge length of about $26 \mathrm{~nm}$. It is worth noting that all the NMNPs were protected by positively-charged CTAB molecules. The capped CTAB bilayer could not only prevent the NPs from aggregation but also play an important role in assembling the NPs into and onto the electrospun nanofibers. The interaction

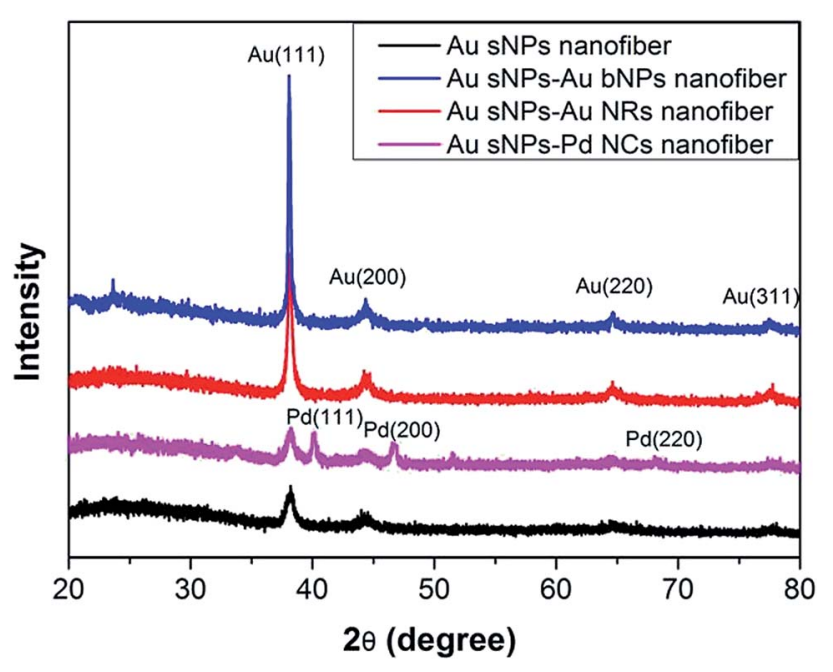

Fig. 3 XRD patterns of the PAA/PVA nanofibers assembled with Au sNPs, Au sNPs-Au bNPs, Au sNPs-Au NRs and Au sNPs-Pd NCs, respectively.

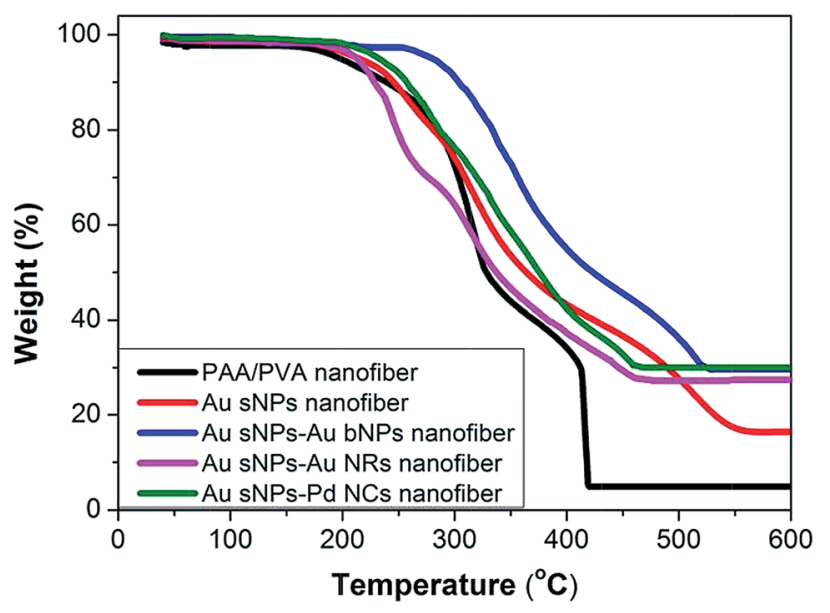

Fig. 4 TG curves of the crosslinked PAA/PVA nanofiber, the PAA/PVA nanofibers assembled with Au sNPs, Au sNPs-Au bNPs, Au sNPs-Au NRs and Au sNPs-Pd NCs. 
between the positively-charged CTAB and the negativelycharged PAA could promote the dispersion of Au NPs in the PAA/PVA solution, which leads to the uniform NP assembly in the as-spun nanofibers. Moreover, the electrostatic interaction is the driving force for the following NP assembly on the PAA/ PVA nanofibers.

In order to obtain relatively uniform nanofibers, the distance between the needle tip and the collector was adjusted. As shown in Fig. $\mathrm{S} 3, \uparrow$ the decrease in needle-to-collector distance leads to an increase in the polydispersity of nanofibers diameters, which might be ascribed to the incomplete evaporation of the solvent and the insufficient bending time of the emitted nanofibers. When the distance was above $25 \mathrm{~cm}$, the relatively uniform nanofibers which could be used to clearly demonstrate the NP assembly were acquired.

Fig. 2 shows the SEM and TEM images of different NMNPs assembled PAA/PVA electrospun nanofibers. Obviously, all these four kinds of NPs were successfully assembled in or on the nanofibers in a relatively uniform fashion. Fig. 2a and b present the distribution of Au SNPs in the PAA/PVA nanofibers, and the $\mathrm{Au}$ sNPs-assembled nanofibers are about $400 \mathrm{~nm}$ in diameter.
Since both PAA and PVA are water-soluble polymers, a thermal treatment was conducted to crosslink the electrospun nanofibers before the assembly of NMNPs onto the nanofibers. ${ }^{15,24,25}$ The esterification reaction (during the thermal treatment) between the carboxylic acid of PAA and the hydroxyl groups of PVA renders the PAA/PVA electrospun nanofibers insoluble in water. After immersing in different NMNPs solution, Au bNPs (Fig. 2c and d), Au NRs (Fig. 2e and f) and Pd NCs (Fig. 2g and h) were successfully assembled on the surface of the Au sNPsassembled PAA/PVA nanofibers. The diameters of these nanofibers changed to about $500 \mathrm{~nm}$ due to the swelling effect of the immersion step. It is noteworthy that there were no significant changes of size and shape of the assembled Au sNPs after the thermal treatment. Hence, NMNPs with different sizes (Au sNPs-Au bNPs), different elemental compositions (Au sNPs-Pd NCs) and different shapes (Au sNPs-Au NRs and Au sNPs-Pd NCs) were facilely assembled both into and onto the PAA/PVA nanofibers. Large area 3D NPs-assembled nanofiber membranes were achieved, as shown in Fig. S2 and S4.† It is anticipated that other functional nanomaterials could also be
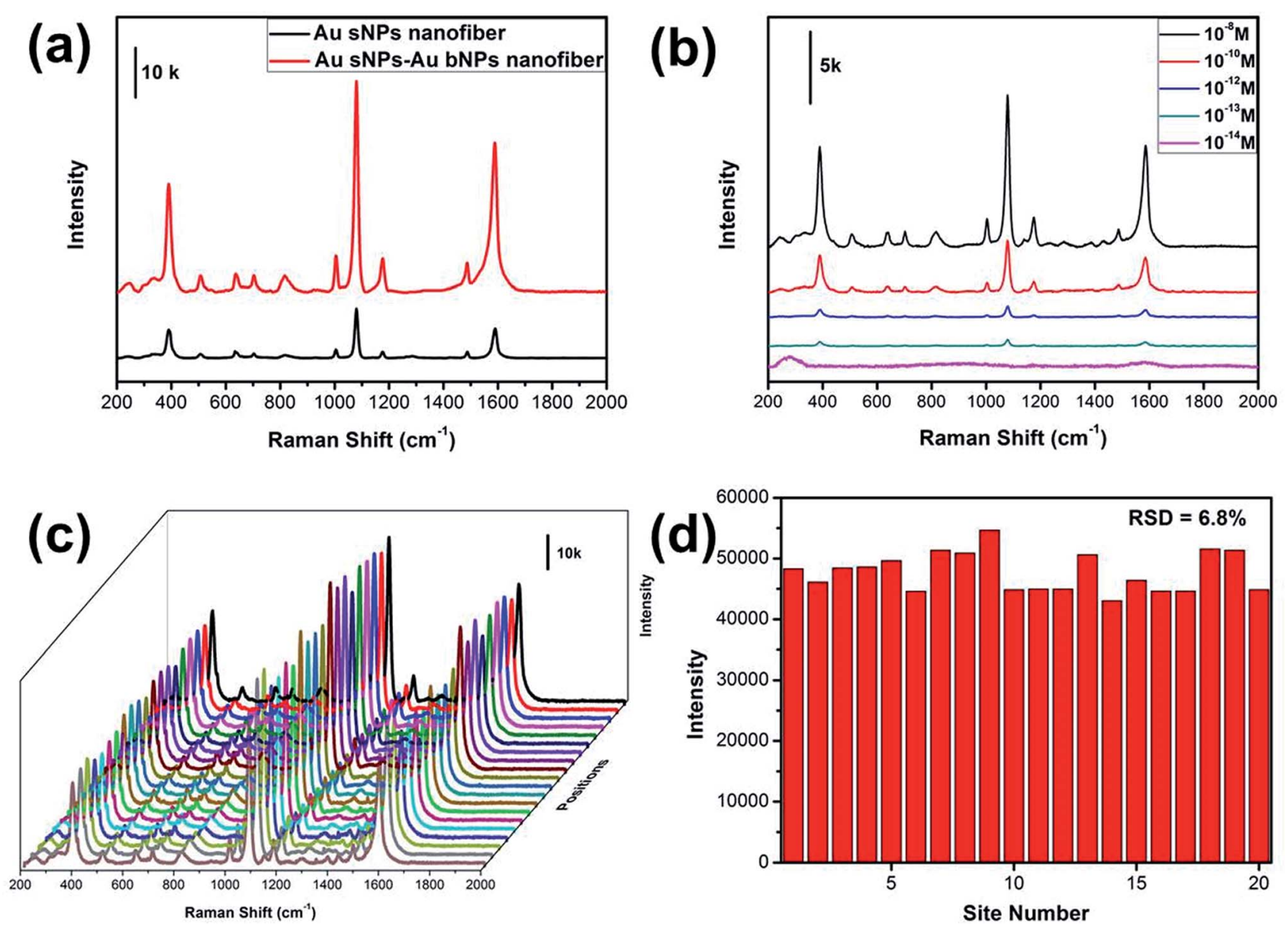

Fig. 5 (a) SERS spectra of $0.1 \mathrm{mM} 4$ 4-ATP molecules collected from the Au sNPs nanofiber and the Au sNPs-Au bNPs nanofiber membrane; (b) SERS spectra of different concentrations of 4-ATP collected from the Au sNPs-Au bNPs nanofiber membrane; (c) SERS spectra of 0.1 mM 4-ATP molecules collected from 20 randomly selected positions of the Au sNPs-Au bNPs nanofiber membrane; (d) the corresponding intensity variation of the $1078 \mathrm{~cm}^{-1}$ peaks in (c). 
assembled with the nanofiber membranes through proper design.

To further confirm the assembly of different NMNPs, XRD as well as TG tests were performed. Compared with the Au sNPsassembled PAA/PVA nanofibers, the Au sNPs-Au bNPs and Au sNPs-Au NRs nanofibers showed enhanced Bragg diffraction peaks of typical face centered cubic Au crystals (Fig. 3), suggesting more $\mathrm{Au}$ nanocrystals were assembled with the electrospun nanofibers after the immersion step. ${ }^{26}$ As for the $\mathrm{Au}$ sNPs-Pd NCs nanofibers, new peaks located at $40.1^{\circ}, 46.5^{\circ}$ and $68.0^{\circ}$ which refer to the (111), (200) and (220) lattice planes of face centered cubic Pd crystals, were observed because of the presence of Pd NCs. ${ }^{21}$ As shown in Fig. 4, the major weight loss occurred from 200 to $550{ }^{\circ} \mathrm{C}$, which might result from the decomposition of the polymers and the small molecules such as CTAB. ${ }^{27}$ The loading content of the assembled Au sNPs inside the nanofibers is $11.5 \mathrm{wt} \%$, while the loading contents of the assembled Au bNPs, Au NRs and Pd NCs outside the nanofibers are $13.2 \mathrm{wt} \%, 11.0 \mathrm{wt} \%$ and $13.5 \mathrm{wt} \%$, respectively. The weight ratios of the organic component and the metal component are estimated to be $7.7: 1,3.0: 1,3.4: 1$ and $3.0: 1$ for the PAA/PVA nanofibers assembled with Au sNPs, Au sNPs-Au bNPs, Au sNPs-Au NRs and Au sNPs-Pd NCs, respectively. Moreover, in order to obtain the exact content of the assembled NPs, the metal content on the nanofibers was measured using inductively coupled plasma optical emission spectrometry. As displayed in Table S2, $\uparrow$ the loading contents for the Au sNPs, Au bNPs, Au NRs and Pd NCs are $10.5 \mathrm{wt} \%, 13.0 \mathrm{wt} \%, 10.5 \mathrm{wt} \%$ and $12.8 \mathrm{wt} \%$, respectively, which agree with the TG results.

It has been well established that the NMNPs-assembled electrospun nanofiber membrane is excellent 3D SERS substrate, which benefits from the numerous "hot spots" generated from the gaps between neighboring NPs. ${ }^{28,29}$ The SERS performance of the NPs-assembled PAA/PVA electrospun nanofiber was evaluated using 4-ATP as the probing molecule. As shown in Fig. 5a, three obvious peaks at 391,1078 and $1578 \mathrm{~cm}^{-1}$ could be assigned to the $\mathrm{a}_{1}$ vibrational modes of 4-ATP, suggesting that the electromagnetic field enhancement dominated the SERS enhancement. ${ }^{30,31}$ Moreover, these characteristic SERS peaks from the $\mathrm{Au}$ sNPs-Au bNPs nanofiber membrane are more intense than those from the Au sNPs nanofiber membrane, which may be ascribed to the increased hot spots. Additionally, the size of the Au bNPs may
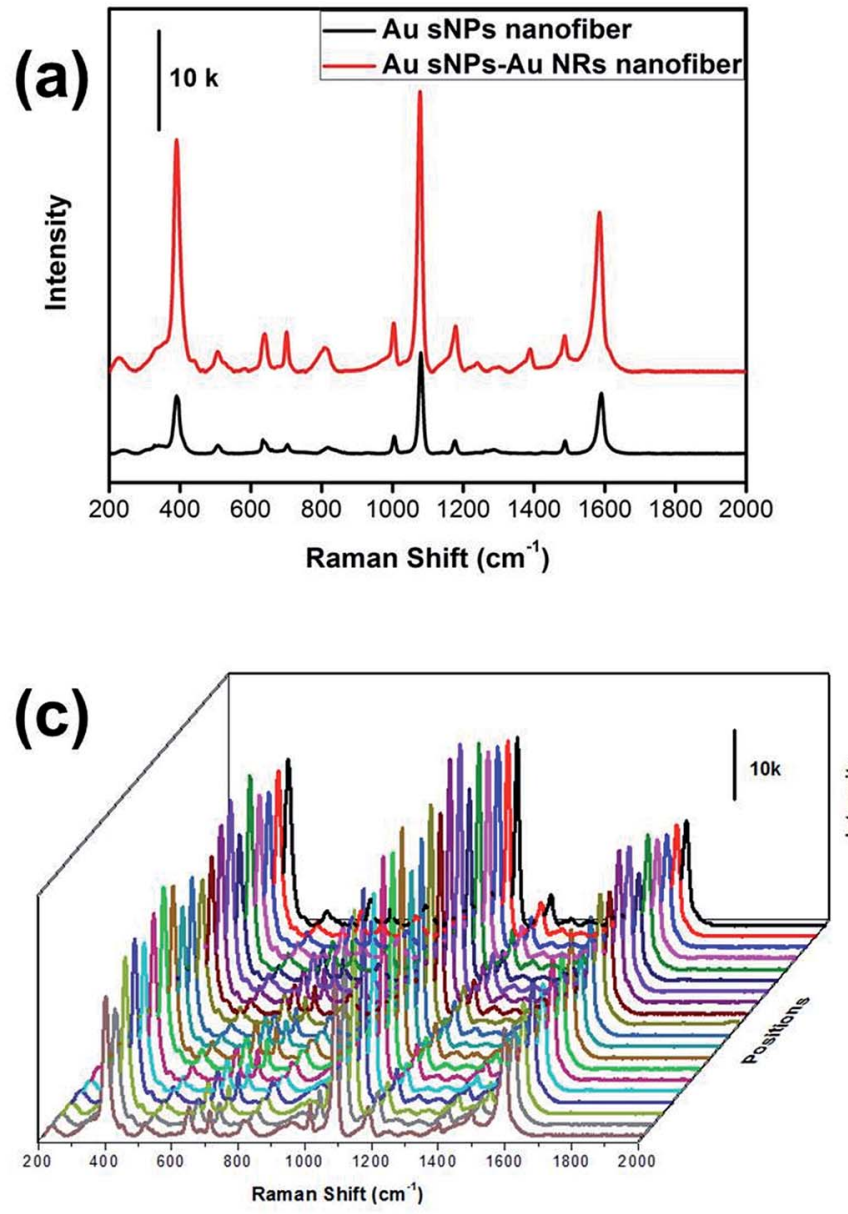
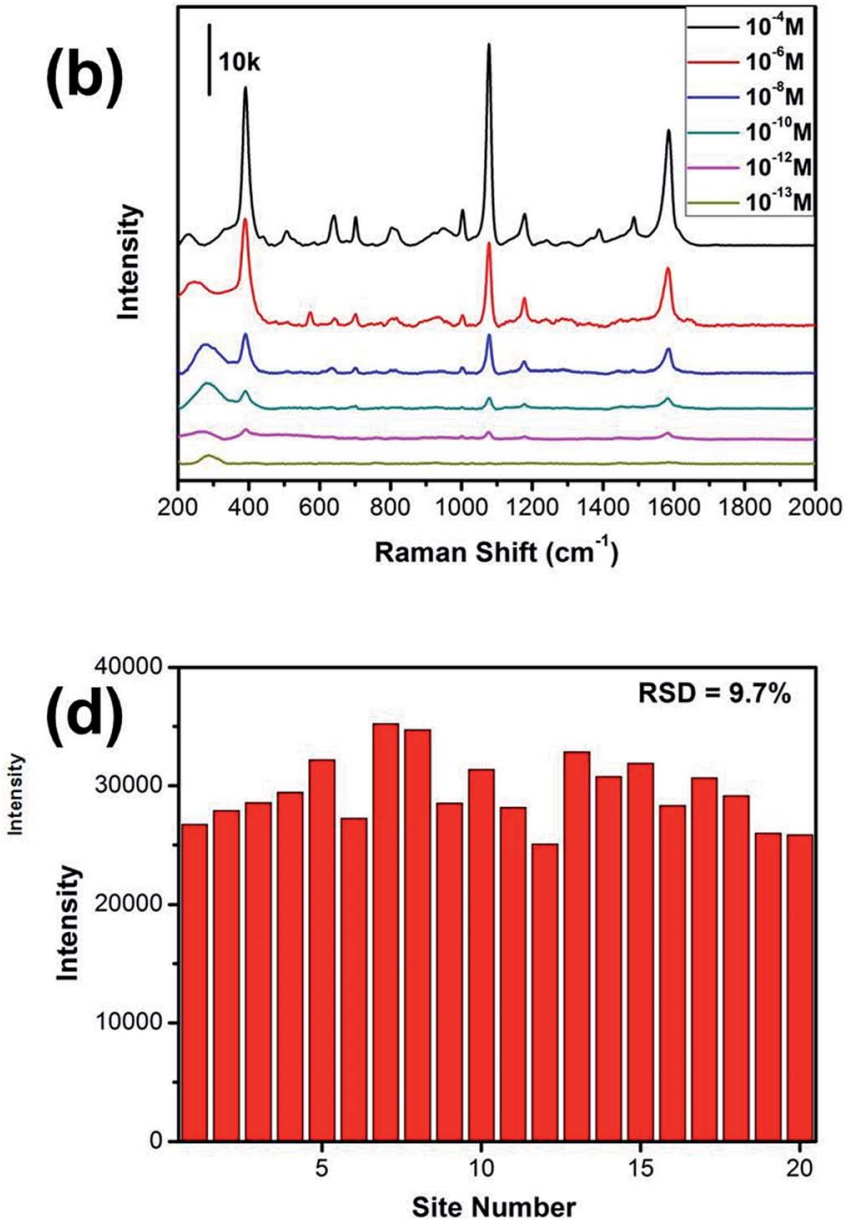

Fig. 6 (a) SERS spectra of $0.1 \mathrm{mM}$ 4-ATP molecules collected from the Au sNPs nanofiber and the Au sNPs-Au NRs nanofiber membrane; (b) SERS spectra of different concentrations of 4-ATP collected from the Au sNPs-Au NRs nanofiber membrane; (c) SERS spectra of 0.1 mM 4-ATP molecules collected from 20 randomly selected positions of the Au sNPs-Au NRs nanofiber membrane; (d) the corresponding intensity variation of the $1078 \mathrm{~cm}^{-1}$ peaks in (c). 
also be responsible for the SERS enhancement. ${ }^{32,33}$ In order to evaluate the actual performance of this 3D SERS substrate (the Au sNPs-Au bNPs nanofiber membrane), the detection of trace amount of 4-ATP was conducted. As shown in Fig. 5b, the SERS intensity decreased with the decreasing concentration of the 4ATP molecule. When the concentration of 4-ATP reached $10^{-13} \mathrm{M}$, the featured peaks at 391,1078 and $1578 \mathrm{~cm}^{-1}$ could be easily located. However, there were no distinguishable peaks in the SERS spectrum when the concentration decreased to $10^{-14} \mathrm{M}$, indicating that the detection limit for the 4-ATP molecule using the Au sNPs-Au bNPs nanofiber membrane was $10^{-13} \mathrm{M}$, which is superior to many similar materials (Table $\mathrm{S} 1 \dagger$ ). The reproducibility of the SERS signals, which is important for the routine analysis, was tested by collecting SERS spectra of 4-ATP from 20 random spots on the Au sNPs-Au bNPs composite nanofibrous membrane (Fig. 5c). There was no obvious difference among these spectra, implying that the reproducible SERS signals are result from the well-distributed NPs. Fig. 5d shows the SERS intensities at $1078 \mathrm{~cm}^{-1}$ of the selected spots in Fig. 5c. The relative standard deviation (RSD) was $6.8 \%$, confirming the excellent reproducibility of the SERS substrate.

Similarly, the SERS performance of the Au sNPs-Au NRs nanofiber membrane was evaluated as well. As expected, the Au SNPs-Au NRs nanofiber membrane exhibited a better SERS performance than the Au sNPs nanofiber membrane (Fig. 6a). The detection limit for the 4-ATP molecule using the Au SNPs$\mathrm{Au}$ NRs nanofiber membrane was $10^{-12} \mathrm{M}$, as displayed in Fig. 6b. Because of the uniform distribution of the Au sNPs and the Au NRs assembled both in and on the PAA/PVA nanofibers, this 3D SERS substrate showed a good SERS reproducibility with a RSD value of $9.7 \%$ (Fig. $6 \mathrm{c}$ and d).

It is known that NMNPs have become promising catalysts for many chemical reactions..$^{34-36}$ Here, the model reduction of 4nitrophenol (4-NP) to 4-aminophenol (4-AP) was utilized to assess the catalytic property of the NPs-assembled electrospun nanofiber membranes. ${ }^{37,38}$ Fig. 7a shows the UV-vis spectra of the 4-NP solution before and after the addition of $\mathrm{NaBH}_{4}$. The characteristic absorption peak shifted from $317 \mathrm{~nm}$ to $400 \mathrm{~nm}$
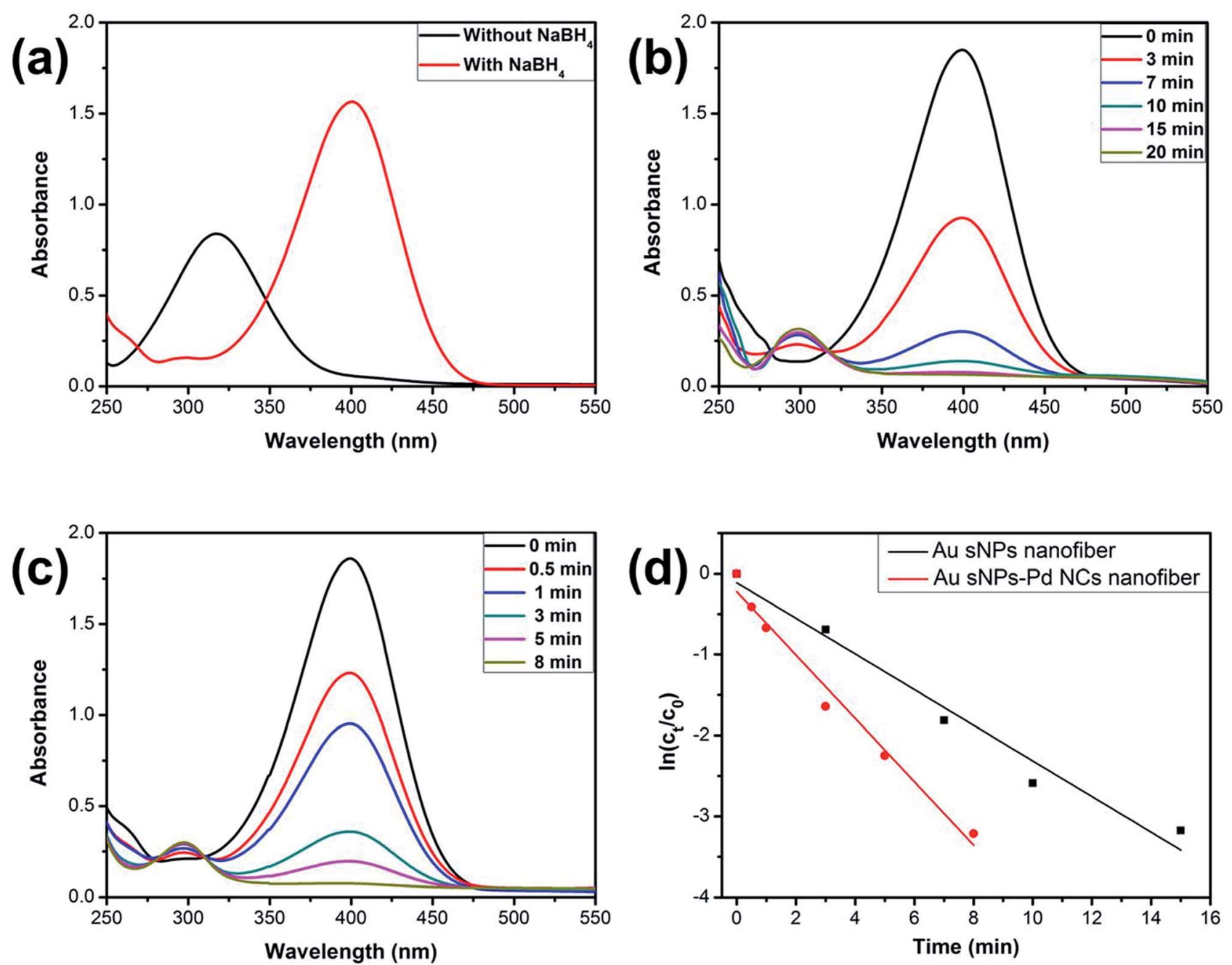

Fig. 7 (a) UV-vis spectra of the 4-NP solution before and after the addition of $\mathrm{NaBH}_{4}$; (b) UV-vis spectra of the catalytic reduction of 4-NP after adding the Au sNPs nanofiber membrane; (c) UV-vis spectra of the catalytic reduction of 4-NP after adding the Au sNPs-Pd NCs nanofiber membrane; (d) plots of $\ln \left(C_{t} / C_{0}\right)$ of $4-N P$ versus time for the reactions using these two kinds of nanocomposite membranes as the catalysts, respectively. 
duo to the formation of 4-nitriphenolate ion after adding $\mathrm{NaBH}_{4} \cdot{ }^{37}$ However, the reduction cannot further proceed without the presence of catalyst. As shown in Fig. $7 \mathrm{~b}$ and $\mathrm{c}$, the absorption peaks at $400 \mathrm{~nm}$, which belong to the 4-nitriphenolate ion, gradually became weaker after the addition of the Au sNPs nanofiber or the Au sNPs-Pd NCs nanofiber membrane as the catalysts. New absorption peaks at $300 \mathrm{~nm}$, which belong to the 4-AP, appeared and increased correspondingly. What is more, the reduction process could be finished within $20 \mathrm{~min}$ and $8 \mathrm{~min}$ for the Au sNPs nanofiber and the Au sNPs-Pd NCs nanofiber membrane, respectively. These results indicate that both the NPs-assembled nanofibrous membranes could efficiently catalyze 4-NP reduction to 4-AP. Since there was excess $\mathrm{NaBH}_{4}$ in the solution, the reaction could be assumed to follow pseudo-firstorder kinetics. Therefore the apparent rate constant $\left(k_{\mathrm{app}}\right)$ could be calculated using the equation $\ln \left(C_{t} / C_{0}\right)=-k_{\text {app }} t$, where the $C_{t}$ and $C_{0}$ are the apparent and initial concentrations of 4-NP, and $t$ represents the reaction time. The calculated values of $k_{\text {app }}$ were $3.7 \times 10^{-3} \mathrm{~s}^{-1}$ and $6.5 \times 10^{-3} \mathrm{~s}^{-1}$ for the Au sNPs nanofiber and the Au sNPs-Pd NCs nanofiber, respectively. This means that the Au sNPs-Pd NCs nanofibrous membrane exhibited better catalytic performance, which could be ascribed to the higher content of the assembled catalytic NMNPs.

\section{Conclusion}

In this work, various NMNPs with different sizes, different elemental compositions and different shapes were successfully assembled both into and onto the PAA/PVA electrospun nanofiber by simple mixing and immersion steps. The NPs were found uniformly distributed in or on the nanofibers. Owing to the increased loading content, the obtained composite nanofibrous membrane assembled with two different kinds of NPs exhibited improved SERS and catalytic performance. Furthermore, this new design strategy and fabrication protocol of the hybrid NPs-assembled nanofiber membrane could be readily extended to the engineering of other functional nanofibrous membrane.

\section{Conflicts of interest}

There are no conflicts to declare.

\section{Acknowledgements}

This work was supported by National Natural Science Foundation of China (Grant 21504083 and 21505123), Shanxi Province Science Foundation for Youths (No. 201601D021035 and 201701D221091) and Program for the Top Young Academic Leaders of North University of China.

\section{References}

1 P. K. Jain, X. H. Huang, I. H. El-Sayed and M. A. El-Sayed, Acc. Chem. Res., 2008, 41, 1578-1586.

2 T. K. Sau and A. L. Rogach, Adv. Mater., 2010, 22, 1781-1804.
3 M. A. Boles, M. Engel and D. V. Talapin, Chem. Rev., 2016, 116, 11220-11289.

4 A. Klinkova, R. M. Choueiri and E. Kumacheva, Chem. Soc. Rev., 2014, 43, 3976-3991.

5 J. Yang, M. K. Choi, D. H. Kim and T. Hyeon, Adv. Mater., 2016, 28, 1176-1207.

6 A. Greiner and J. H. Wendorff, Angew. Chem., Int. Ed., 2007, 46, 5670-5703.

7 W. E. Teo and S. Ramakrishna, Nanotechnology, 2006, 17, R89-R106.

8 C. L. Zhang and S. H. Yu, Mater. Horiz., 2016, 3, 266-269.

9 S. Agarwal, A. Greiner and J. H. Wendorff, Prog. Polym. Sci., 2013, 38, 963-991.

10 D. He, B. Hu, Q. F. Yao, K. Wang and S. H. Yu, ACS Nano, 2009, 3, 3993-4002.

11 C. L. Zhang, K. P. Lv, H. P. Cong and S. H. Yu, Small, 2012, 8, 648-653.

12 W. Gao, G. Chen, W. Xu, C. Yang and S. Xu, RSC Adv., 2014, 4, 23838-23845.

13 J. H. Park and Y. L. Joo, Soft Matter, 2014, 10, 3494-3505.

14 Y. W. Qian, G. W. Meng, Q. Huang, C. H. Zhu, Z. L. Huang, K. X. Sun and B. Chen, Nanoscale, 2014, 6, 4781-4788.

15 T. Yang, J. Ma, S. J. Zhen and C. Z. Huang, ACS Appl. Mater. Interfaces, 2016, 8, 14802-14811.

16 P. Jia, B. Cao, J. Q. Wang, J. Qu, Y. X. Liu and K. Pan, Analyst, 2015, 140, 5707-5715.

17 W. Tang, D. B. Chase and J. F. Rabolt, Anal. Chem., 2013, 85, 10702-10709.

18 H. Zhu, M. L. Du, M. Zhang, P. Wang, S. Y. Bao, M. L. Zou, Y. Q. Fu and J. M. Yao, Biosens. Bioelectron., 2014, 54, 91-101.

19 C.-L. Zhang, K.-P. Lv, H.-T. Huang, H.-P. Cong and S.-H. Yu, Nanoscale, 2012, 4, 5348-5355.

20 N. R. Jana, L. Gearheart and C. J. Murphy, Langmuir, 2001, 17, 6782-6786.

21 X. S. Shen, G. Z. Wang, X. Hong and W. Zhu, Crystengcomm, 2009, 11, 753-755.

22 B. Nikoobakht and M. A. El-Sayed, Chem. Mater., 2003, 15, 1957-1962.

23 C. Noguez, J. Phys. Chem. C, 2007, 111, 3806-3819.

24 S. L. Xiao, M. W. Shen, R. Guo, Q. G. Huang, S. Y. Wang and X. Y. Shi, J. Mater. Chem., 2010, 20, 5700-5708.

25 D. M. Hu, Y. P. Huang, H. Liu, H. Wang, S. G. Wang, M. W. Shen, M. F. Zhu and X. Shi, J. Mater. Chem. A, 2014, 2, 2323-2332.

26 S. J. H. Fathima, J. Paul and S. Valiyaveettil, Small, 2010, 6, 2443-2447.

27 S. L. Xiao, M. W. Shen, R. Guo, S. Y. Wang and X. Y. Shi, J. Phys. Chem. C, 2009, 113, 18062-18068.

28 J. D. Shao, L. P. Tong, S. Y. Tang, Z. N. Guo, H. Zhang, P. H. Li, H. Y. Wang, C. Du and X. F. Yu, ACS Appl. Mater. Interfaces, 2015, 7, 5391-5399.

29 C. H. Lee, L. M. Tian, A. Abbas, R. Kattumenu and S. Singamaneni, Nanotechnology, 2011, 22, 275311.

30 N. Takeyasu, R. Kagawa, K. Sakata and T. Kaneta, J. Phys. Chem. C, 2016, 120, 12163-12169. 
31 Y. F. Huang, D. Y. Wu, H. P. Zhu, L. B. Zhao, G. K. Liu, B. Ren and Z. Q. Tian, Phys. Chem. Chem. Phys., 2012, 14, 84858497.

32 P. P. Fang, J. F. Li, Z. L. Yang, L. M. Li, B. Ren and Z. Q. Tian, J. Raman Spectrosc., 2008, 39, 1679-1687.

33 V. Joseph, A. Matschulat, J. Polte, S. Rolf, F. Emmerling and J. Kneipp, J. Raman Spectrosc., 2011, 42, 1736-1742.
34 M. A. Shenashen, S. A. El-Safty and E. A. Elshehy, Part. Part. Syst. Charact., 2014, 31, 293-316.

35 C. H. Cui and S. H. Yu, Acc. Chem. Res., 2013, 46, 1427-1437.

36 R. Narayanan, Molecules, 2010, 15, 2124-2138.

37 T. Aditya, A. Pal and T. Pal, Chem. Commun., 2015, 51, 94109431.

38 P. Herves, M. Perez-Lorenzo, L. M. Liz-Marzan, J. Dzubiella, Y. Lu and M. Ballauff, Chem. Soc. Rev., 2012, 41, 5577-5587. 